\title{
Chyluria Associated with Nephrotic- Range Proteinuria: Pathophysiology, Clinical Picture and Therapeutic Options
}

\author{
Giorgio Graziani $^{a}$ David Cucchiari ${ }^{a}$ Simona Verdesca ${ }^{a} \quad$ Luca Balzarini $^{b}$ \\ Alessandro Montanellic Claudio Ponticelli ${ }^{\mathrm{a}}$ \\ ${ }^{a}$ Nephrology and Dialysis Unit, ${ }^{b}$ Magnetic Resonance Section, Department of Radiology, and 'Biochemical and \\ Clinical Laboratory, Istituto Clinico Humanitas IRCCS, Rozzano, Italy
}

\section{Key Words}

Chyluria - Lymphangioma - Medium-chain triglycerides •

Nephrotic proteinuria

\begin{abstract}
Chyluria denotes the urinary excretion of chyle, which is a lymphatic fluid rich in chylomicrons. Chyle flows from the intestinal lacteals to the left subclavian vein through the thoracic duct. When an abnormal connection between these structures and the urinary tract develops, chyluria appears. The syndrome is often associated with a nephrotic-range proteinuria, and this could be a wrong indication to perform renal biopsy. Chyluria is classified as parasitic or nonparasitic, the former being induced by lymphatic filariasis, whereas the latter is caused by medical, traumatic or inherited diseases. The patient usually reports excretion of milky urines, monolateral flank pain, malnutrition, weight loss and weakness. Urinalysis demonstrates lymphocyturia associated with chylomicrons and triglycerides in the supernatant. The diagnostic approach is aimed to define the site of lymphourinary fistula. A selective ureteral catheterization allows to
\end{abstract}

collect urine samples from each kidney, demonstrating a monolateral source of proteins and lipids and making renal biopsy superfluous. Other diagnostic tools include nuclear magnetic resonance urography and lymphoangiography. Many therapeutic options have been proposed. Sclerosing solution instillation into the renal pelvis and laparoscopic renal pedicle disconnection are the invasive procedures most commonly employed. Among the medical alternatives, a low-fat diet supplemented with medium-chain triglycerides is often followed by complete clinical and biochemical remission.

Copyright $\odot 2011$ S. Karger AG, Basel

Chyluria is the presence of chyle in the urine. Chyle is a milky fluid composed of lymph and absorbed dietetic fats that are assembled as chylomicrons, taken up by lymph vessels, conveyed to the thoracic duct, and then drained into the left subclavian vein. In healthy subjects, the lymphatic vessels do not communicate with the urinary tract. Whenever there is an abnormal communication between these two structures, chyle leaks into the

\section{KARGER}

Fax +41613061234 E-Mail karger@karger.ch www.karger.com
(C) 2011 S. Karger AG, Basel

$1660-2110 / 11 / 1193-0248 \$ 38.00 / 0$

Accessible online at:

www.karger.com/nec
Prof. Giorgio Graziani

Istituto Clinico Humanitas IRCCS

Via Manzoni 56

IT-20089 Rozzano (Italy)

Tel. +39 028224 4567, E-Mail giorgio.graziani@humanitas.it 
urine and the emulsionated chylomicrons give it a typical milky and cloudy appearance which is often one of the first signs reported by the patient.

\section{Etiology and Pathogenesis}

Chyluria is usually classified as parasitic or nonparasitic on the basis of its etiopathogenesis (table 1). While parasitic forms are almost exclusively responsible for cases detected in Eastern countries, the other nonparasitic forms are more frequent in Western countries.

The most common cause of parasitic chyluria is lymphatic filariasis that is caused by three nematodes: Wuchereria bancrofti, which accounts for $90 \%$ of infections, Brugia malayi and Brugia timori. It is estimated that about 120 million people in 83 countries in SouthEast Asia, India, Pacific Islands, Africa and, to a lesser extent, South and Central America, are infected [1]. Filariasis classically causes massive limb lymphedema, a condition called 'elephantiasis', and scrotal swelling in men, while the occurrence of chyluria is an unusual manifestation of a long-standing disease. Other parasitic forms include cysticercosis, echinococcosis, malaria and ascariasis [2].

Causes other than parasitic include traumatic factors, infections, tumors and inherited conditions. Traumatic lesions leading to chyluria mainly result from surgical procedures, more often in patients submitted to partial nephrectomy or nephron-sparing surgery for renal cell carcinoma. Chyluria can also be caused by other infections, namely those associated with granulomatous reaction: tuberculosis, leprosy and mycosis. Pregnancy and aortic aneurysm can occasionally cause lymphatic obstruction leading to the development of the syndrome. In neoplastic patients, chyluria may be caused by extrinsic compression or by neoplastic cells spreading into lymphatics resulting in their swelling and rupture.

Rarely, chyluria is caused by an inherited lymphangioma which is an innate variceal malformation of lymphatic vessels promoting a lymphourinary communication.

Whatever the cause, if the lymphatic drainage from the bowel is obstructed, lymphatic vessels dilate and can break and flood into the urinary tract. Because of the strict anatomical connection of retroperitoneal lymphatic ducts with the peripelvic and periforniceal ones [3], the calyceal fornix is the most frequent site of a lymphourinary fistula. Less frequently, the lymphatic channels may open at ureteral or bladder sites.

Chyluria and Nephrotic Proteinuria
Table 1. Most common causes of chyluria

\begin{tabular}{ll}
\hline Parasitic causes & Nonparasitic causes \\
\hline Lymphatic filariasis & Traumatic lesions (surgery) \\
Cysticercosis & Tumors \\
Echinococcosis & Malformations (lymphangioma) \\
Malaria & Pregnancy \\
Ascariasis & Aortic aneurysm \\
& Granulomatous infections \\
\hline
\end{tabular}

\section{Clinical Picture}

The excretion of milky or cloudy urine is the principal sign of chyluria. This characteristic urinary picture is due to the emulsionated chylomicrons coming from intestinal lacteals. The excretion of milky urine is characteristically observed after a fatty meal while it can disappear in a fasting condition. The clinical onset of chyluria is frequently characterized by monolateral flank pain resembling renal colic, likely induced by the transit or retention of ureteral clots. This complaint usually occurs at night, obliging the patient to a continuous use of analgesics. The renal function is generally normal. Spontaneous remission of chyluria frequently occurs in about $50 \%$ of cases [4]; however, in those patients where it persists, due to continuous urinary loss of fats, proteins, lymphocytes and fat-soluble vitamins, a delayed diagnosis can lead to severe malnutrition, dehydration, immunodysfunction and hypercoagulability $[5,6]$. Patients complain of weight loss, weakness and mental depression with severe impairment of their quality of life. Moreover, chyluria is often associated with a nephroticrange proteinuria due to the high protein concentration of chyle. At physical examination, blood pressure is usually low-normal, due to fluid depletion, and pitting edema is more evident when the patient is severely malnourished and hypoalbuminemic.

On the other hand, hypocholesterolemia and hypotriglyceridemia caused by the abnormal urinary fat excretion can differentiate the classical nephrotic syndrome from the proteinuria and hypoproteinemia induced by chyluria [7].

In this context, the most useful diagnostic tool is urine sediment analysis which allows to differentiate all the other causes of cloudy and milky urine from chyluria and possibly lead the nephrologist to suspend the execution of renal biopsy in order to perform more accurate investigations (table 2).

Nephron Clin Pract 2011;119:c248-c254 
Urine gross examination reveals a muddy and whitish aspect, with gelatinous and unctuous clots. Macroscopic inspection shows that cloudy or milky urine remains turbid also after centrifugation, while after standing, it separates into three layers: chylomicrons on the top, proteins in the middle and fibrin at the bottom [7]. The addition of Sudan III to the specimen demonstrates a superficial layer of red-stained fatty particles over clear urine after resting. Adding an equal volume of chloroform to the urine, after shaking and resting, separates the specimen into two layers: milky on the surface and clear below. The absence of fatty droplets, fatty casts and renal tubular epithelial cell casts, that are hallmarks of nephrotic sediment, can easily differentiate chyluria from nephrotic syndrome. Moreover, when microhematuria is present in patients with chyluria, it is always isomorphic in spite of a dysmorphic erythrocyturia in nephrotic syndrome.

In patients with chyluria, the urinary sediment is characterized by lymphocyturia. A nephrologist expert in urinary sediment analysis can identify lymphocytes by contrast-phase microscopy. The presence of lymphocytes can be also assessed using specific stains (e.g. Papanicolau stain) [8].

Moreover, macroscopic appearance of cloudy urine should always raise suspicion of massive crystalluria, a huge amount of squamous epithelial cells (genital contamination) and urinary tract infections.

In the first two cases, a careful analysis of urinary sediment reveals the presence of crystals or epithelial cells. In urinary tract infections, the presence of neutrophils (in absence of lymphocyturia) and bacteriuria allows to differentiate this condition from chyluria.

\section{Diagnosis}

To diagnose filariasis, detection of microfilariae in blood smear is a specific and inexpensive test; however, it is uncomfortable because it should be performed at night, and it lacks sensitivity. Nowadays, the best diagnostic option is the filarial antigen test performed with immunochromatographic card tests. This technique is more sensitive than blood smear, but it does not detect Brugia infections. Antibody detection can overcome this limitation as it is sensitive to Brugia; however, this test has not been widely employed because antigen testing is more convenient [9].

After exclusion of parasitic etiology, which requires a specific antiparasitic therapy, in every case of chyluria the major objective is to localize the site of lymphourinary
Table 2. Main clinical and laboratory differences between chyluria and nephrotic syndrome

\section{Chyluria \\ Nephrotic syndrome}

Clinical features

\begin{tabular}{lll} 
Edema & $+/-$ & +++ \\
Flank pain and renal colic & +++ & - \\
Hypoalbuminemia & $+/-$ & +++ \\
Hypercholesterolemia & - & +++ \\
\hline
\end{tabular}

Time course

Onset

Episodic and Gradual dependent on fatty intake

Persistence

Urine gross examination

Cloudy and milky

Muddy and whitish

Unctuous clots

Sudan III

$-$

$++$

+++
+++
+++
Red-stained layer
on the surface
containing fats

\section{Urine sediment}

Lymphocytes

Microhematuria

Pathological casts

Lipiduria

+++
++
Isomorphic
-
-

$+++$

-
+
+
Dysmorphic
+++
+++
Oval fat bodies
and lipid droplets

fistula in order to choose the best therapeutic approach for each patient. For this purpose, the first imaging technique is renal ultrasonography that may allow to recognize a unilateral calico-pyelic dilation, secondary to ureteral clot obstruction and a hypodense fat-fluid level supernatant in the bladder. Ultrasonography may also show peripelvic or periureteral dilated lymphatic vessels, which helps to identify the site of lesion. The urological workup is followed by flexible cystoscopy that confirms the presence of gelatinous urine and adipose fragments in the bladder. It also allows to identify the ureteral papilla from which whitish urine is ejaculated. Cystoscopy is followed by selective ureteral catheterization to collect urine samples from each kidney. A chemical analysis of these samples demonstrates which kidney sustains proteinuria and lypiduria by measuring the amount of proteins, triglycerides and cholesterol excreted separately. By demonstrating the unilateral origin of urinary protein and lipid excretion, glomerular nephropathy can be excluded, useless 
renal biopsy can be avoided and attention can be turned to a monolateral urological pathology. Subsequently, monolateral ureteroscopy provides endoluminal sonographic images for diagnostic needs and helps to choose the best therapeutic option. However, the identification of lymphourinary fistula site, although very important, neither provides a complete overview of the abdominal lymphatics nor clarifies the nature and the pathogenesis of lymphatic vessel dilation and rupture, meaning that other diagnostic tools are indicated.

Contrast-enhanced CT scan images are of little help because the intravenous iodine contrast does not show lymphatic vessels.

MRI with its higher soft tissue contrast resolution is more sensitive in detecting retroperitoneal pathologies. In particular, magnetic resonance 'urography' obtained with T2W fat-sat sequences may show local or extensive variceal ectasia of the lomboaortic lymphatic ducts and its connection with the urinary apparatus, allowing to identify the site and the etiology of lymphourinary fistula. However, lymphography with bipedal incannulation of lymphatic vessels is considered the gold standard technique to demonstrate pathological alterations of lymphatic vessels (fig. 1) [10].

\section{Therapy}

Anthelmintic drugs are mandatory when parasitic etiology is confirmed. Drugs commonly used are diethylcarbamazine, ivermectine and albendazole. In the year 2000 , the WHO launched a global program with the aim to eliminate lymphatic filariasis by 2020 from those countries where it is endemic, by means of a chemotherapy that prevents human-to-vector transmission [1].

Subsequently, in every case of chyluria, treatment is chosen based on the severity of the clinical picture. The high rate of spontaneous remission may suggest conservative treatment rather than an invasive procedure in patients with mild disease.

However, even before choosing the therapeutic option, the same lymphoangiography can be effective due to the sclerosing properties of the contrast agent. Thus, this apparently out-of-date diagnostic tool can be useful not only to assess where the lymph leaks, but also to treat this kind of patients. Yamagami et al. [11] hypothesized the mechanism involved. They observed that the dye accumulates at the site of fistula and does not advance beyond that point, resulting in an inflammatory reaction that finally leads to the fibrotic obstruction of lymph flow.

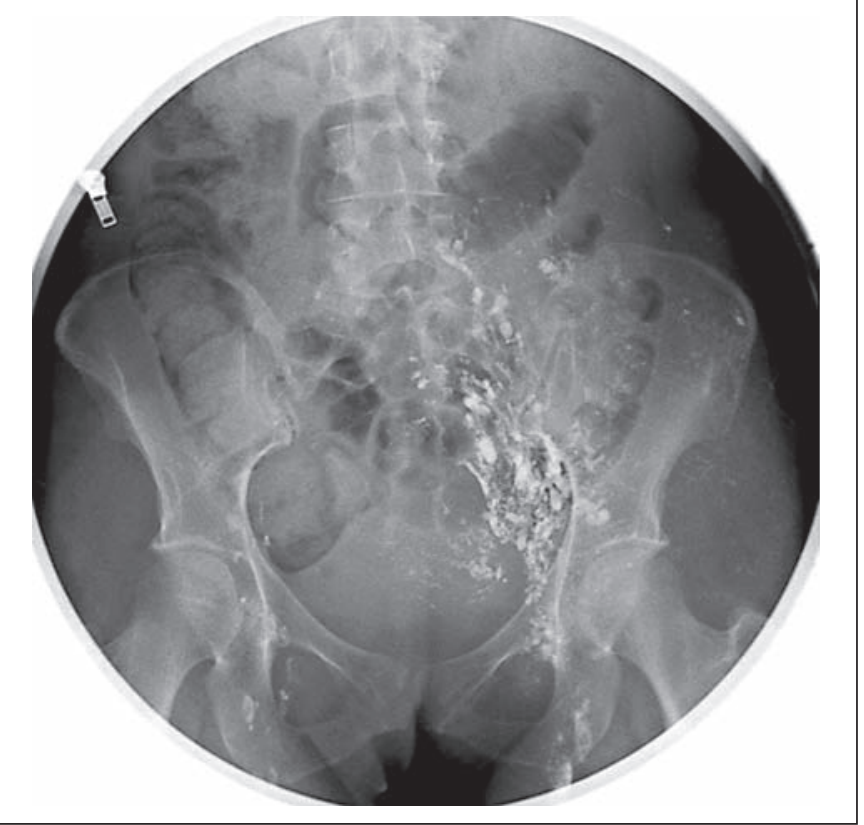

Fig. 1. Lymphography with bipedal incannulation of lymphatic vessels shows an inherited variceal dilation of the lymphatic tree in the left pelvic region. The so-called lymphangioma is a cause of nonparasitic permanent chyluria.

Among the conservative treatments, intravenous infusion of somatostatin or subcutaneous administration of its synthetic analogue, octreotide acetate [12] can improve the lipid homeostasis and reduce lymph flow along the thoracic duct.

The use of ACE inhibitors is an anecdotal medical option reported by Saleh [13] who hypothesized an action of this drug class on the lymph circulation in the kidney.

Long-chain triglyceride avoidance in the diet is an effective treatment to reduce chyluria [14]. In this nutritional setting, chylomicrons are no longer produced by the enterocytes and chyluria stops. However, this diet therapy leads to severe malnutrition, weakness and weight loss.

To contrast these side effects, a medium-chain triglyceride (MCT) oil is recommended. Instead of normal lipids, which are assembled in chylomicrons and delivered to thoracic duct, MCTs are rapidly absorbed from the gut and directly transported to the liver via portal vein, bypassing the lymphatic circulation. They are stored in the liver and metabolized mostly as chetons providing many calories to contrast malnutrition, weight loss and immunodysfunction.

This therapeutic approach was first proposed many years ago and, surprisingly, although presenting many 
Fig. 2. Algorithm for the diagnosis and

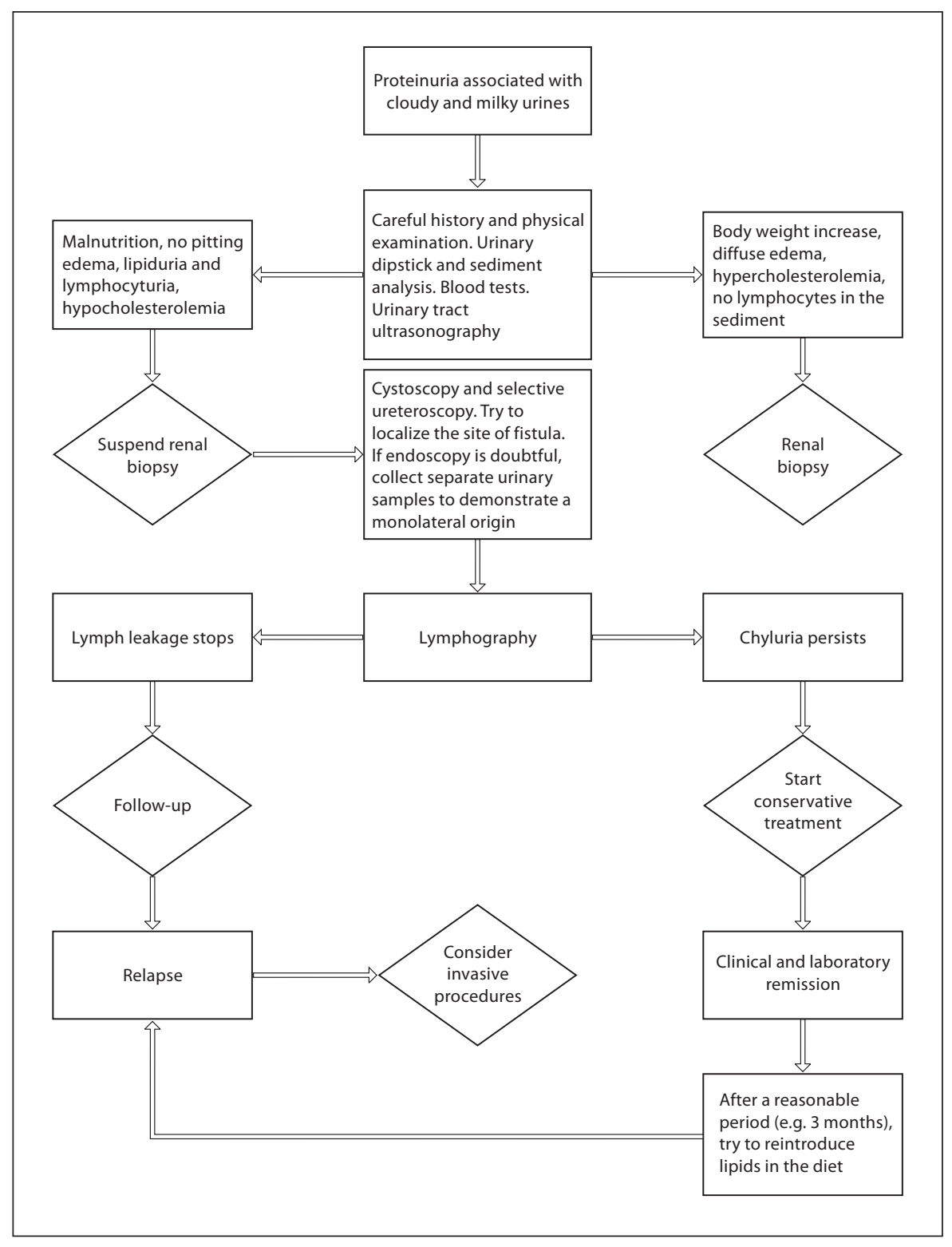
treatment of chyluria.

advantages, it has not been extensively employed as an alternative noninvasive therapy. In our recent experience, this diet schedule was performed on a young girl presenting long-lasting chyluria due to inherited lymphangioma. This patient was treated for 4 years with impressive results: chyluria completely reversed, the patient gained weight and significantly improved her quality of life, without any significant side effect [unpubl. data]. In this context, another important issue is that the long-lasting lymph transit stop could have induced closure of the fistula with non-invasive resolution of the syndrome.
In many patients, a more aggressive approach could be indicated when symptoms (renal colics) and complications (malnutrition, immunodysfunction) lead to a poor quality of life or when conservative treatment does not achieve remission. A rationale and complete diagnostic and therapeutic algorithm is shown in figure 2 .

Invasive approaches include endoscopic and surgical procedures. Sclerosing solution (e.g. silver nitrate) instillation into the renal pelvis has a relatively high success rate (about $80 \%$ ) and is the technique most commonly employed [15]. Nevertheless, the recurrence of chyluria, papillary necrosis, renal and hepatic failure and necrotiz- 
ing ureteritis can be severe and even life-threatening adverse events $[16,17]$. An alternative endoscopic option recently proposed is the topical application of neodymiumYAG laser [18].

The surgical procedure of choice is renal pedicle disconnection which can be performed with either laparoscopy or open surgery and leads to remission of chyluria in most patients. Zhang et al. [19] demonstrated the same effectiveness of laparoscopy compared to traditional surgery, with the advantages of minimal invasion, shorter postoperative hospital stay and a more rapid recovery.

Other surgical options include lymphovenous anastomosis and autotransplantation, but nowadays the best treatment available for severe cases of chyluria is laparoscopic renal pedicle disconnection.

\section{Conclusions}

When a nephrotic-range proteinuria is accompanied by the atypical clinical picture described above, nephrologists should keep in mind the possibility of chyluria. Macroscopic milky appearance of urine, a simple urinary sediment analysis and the absence of typical hematological signs of nephrotic syndrome can confirm the clinical suspicion. Thereafter, when a urological workup demonstrates a monolateral source of lipids and proteins, renal biopsy should be definitively suspended, and the diagnostic algorithm should be turned towards locating the site of lymphourinary fistula. The apparently outdated lymphoangiography is the best diagnostic tool in this setting. Patients who have just mild disease may benefit from long-term conservative treatment based on a low-fat diet supplemented with MCT oil, since spontaneous remissions are frequent and other invasive therapeutic options carry the risk of severe complications.

Therefore, a current working hypothesis could be to attempt this dietetic schedule in patients with severe disease, reserving more invasive procedures only for those patients who do not respond to the diet therapy.

\section{Disclosure Statement}

The authors declare no conflict of interest.

\section{References}

1 Taylor MG, Hoerauf A, Bockarie M: Lymphatic filariasis and onchocerciasis. Lancet 2010;376:1175-1185.

-2 Gulati S, Gupta N, Singh NP, Batra S, Garg S, Beniwal P, Kumar S: Chyluria with proteinuria or filarial nephropathy? An enigma. Parasit Int 2007;56:251-254.

3 Chen KC: Lymphatic abnormalities in patients with chyluria. J Urol 1971;106:111-114.

4 Ohyama C, Saita H, Miyasato N: Spontaneous remission of chyluria. J Urol 1979;121: 316-317.

5 Date A, John TJ, Chandy KG, Rajagopalan MS, Vaska PH, Pandey AP, Shastry JC: Abnormalities in the immune system in patient with chyluria. Br J Urol 1981;53:384-386.

6 Golestaneh L, Karam S, Lawrence J, Yang S, Greenberg M: Hypercoagulability in a patient with chronic chyluria, proteinuria and hypoalbuminaemia. Nephrol Dial Transplant 2009;24:3240-3243.

7 Cheng J-T, Mohan S, Nasr SH, D'Agati VD: Chyluria presenting as milky urine and nephrotic-range proteinuria. Kidney Int 2006; 70:1518-1522.
8 Neuwirt H, Eller P, Tiefenthaler M, Mayer G, Rosenkranz AR: A rare case of milky urine. Wien Klin Wochenschr 2010;122:596-600.

9 Weil GJ, Ramzy RM: Diagnostic tools for filariasis elimination programs. Trends Parasitol 2007;23:78-82.

10 Sharma S, Hemal AK: Chyluria - an overview. Int J Nephrol Urol 2009;1:14-26.

- 11 Yamagami T, Masunami T, Kato T, Tanaka O, Hirota T, Nomoto T, Mikami K, Miki T, Nishimura T: Spontaneous healing of chyle leakage after lymphangiography. Br J Radiol 2005;78:854-857.

12 Giordano M, Cirillo D, Baron I, Sorrentino F, Tomasino G, Cicchella T, De Bellis L, Giordano C: Treatment of post-traumatic chyluria with sub-cutaneous octreotide administration. Nephrol Dial Transplant 1996;11:365-367.

13 Saleh AE: Angiotensin-converting-enzyme inhibitors and chyluria. Ann Intern Med 1993;119:1223-1224.

14 Hashim S, Roholt HB, Babayan VK, van Itallie TB: Treatment of chyluria and chylothorax with medium chain triglyceride. N Engl J Med 1964;270:756-761.
15 Dhabalia JV, Pujari NR, Kumar V, Punia MS, Gokhale AD, Nelivigi G: Silver nitrate sclerotherapy for chyluria: evaluation of the optimal instillation regimen. Urol Int 2010; 85:56-59.

16 Kulkarni AA, Pathak MS, Sirsat RA: Fatal renal and hepatic failure following silver nitrate instillation for treatment of chyluria. Nephrol Dial Transplant 2005;20:12761277 .

17 Su CM, Lee YC, Wu WJ, Ke HL, Chou YH, Huang $\mathrm{CH}$ : Acute necrotizing uretritis with obstructive uropathy following instillation of silver nitrate in chyluria: a case report. Kaohsiung J Med Sci 2004;20:512-515.

18 Lenggenhager C, Warzinek T, Wüthrich RP, Müller J, Schmid HP, Engeler DS: Nonparasitic chyluria treated by laser. Urologe 2007; 46:1715-1717.

19 Zhang X, Zhu QG, Ma X, Zheng T, Li HZ, Zhang J, Fu B, Lang B, Xu K, Pan TJ: Renal pedicle lymphatic disconnection for chyluria via retroperitoneoscopy and open surgery: report of 53 cases with followup. J Urol 2005; 174:1828-1831. 


\section{Editorial Comment}

Meguid El Nahas, Sheffield

The mini-review by Graziani et al. reminds nephrologists of the diagnostic and therapeutic approaches to patients with milky urine secondary to chyluria. This up-todate review discusses parasitic, most commonly filariasis, causes of chyluria in the tropics but also more common causes in the West such as lymphangioma, carcinoma, trauma, abscess, tuberculosis, pregnancy and stenoses of the thoracic duct. Many such causes generate lymphourinary communications leading to chyluria. Distinction between nephrotic syndrome and chyluria, both associated with heavy proteinuria, can easily be made by urine analysis; the cloudy/milky aspect, lymphocyturia as well as the positive reaction to Sudan III characterize chyluria. Also, hypocholesterolemia in chyluria contrasts with the hyperlipidemia of nephrotic syndrome. Preferred investigations include MRU and lymphangiography as well as MR lymphography. Clearly, treatment is guided by the underlying cause ranging from antiparasitic antibiotics to sclerosing therapy. The authors also put forward a conservative, dietary, therapeutic approach consisting of a low-fat diet supplemented with medium-chain triglycerides. Such an approach may be worth considering in selected patients before embarking on more invasive approaches. Chyluria remains an uncommon renal manifestation but one that warrants careful investigation to exclude more sinister underlying pathologies. 